\title{
La confection des tableaux de bord : réflexions préliminaires sur l'évaluation de l'efficacité de l'action financière des agences de l'eau
}

\author{
Indicator schedule making : preliminary reflection about the \\ efficiency assessment of the french water agencies financial action \\ par Guy Bédiot \\ Agence de l'eau Seine-Normandie
}

The efficiency assessment of the french water agencies financial action on water environment and public health is not only a simple technical problem as " how to convert elementary data into indicator ?". Actually this question is quickly supplanted by the organisational one "who are the participants involved and on which agreement are they ready to come? ". But the policy management will define each organisation's way of working, which is guided by its own interest. The complete innocent thinks the general interest should federate the action at every level: that's not the case because the final objectives are often contradictory. A few examples will show that a single indicator schedule is not relevant and that the lack of scientific knowledge is the cause of difficulties.

Depuis le début de son VIème programme quinquennal (1992-1996), l'agence de l'eau Seine-Normandie se trouve confrontée à un débat relatif à l'évaluation de l'impact de la politique des agences de l'eau : derrière cette expression se cachent plusieurs interprétations de ce qu'est une évaluation. L'objet de cet article est d'apporter quelques éclairages sur un sujet difficile, délicat et controversé. Sans ignorer ce dernier aspect, il est proposé de partir de la demande des présidents de comité de bassin et de conseil d'administration des 6 agences de l'eau exprimée lors de la conférence de l'automne 1995 et concernant la réalisation d'indicateurs d'efficacité de l'action financière des agences de l'eau sur le milieu et la santé publique. "Il faut se fixer comme objectif (ce qui sera difficile) de mettre en lumière le lien entre les ouvrages financés et l'effet sur le milieu " : cet extrait du compte rendu de la conférence précitée montre que les 12 présidents sont conscients de la difficulté, mais veulent obtenir des réponses. La figure 1 schématise la demande des présidents [1].

Plusieurs voies s'offrent à nous pour répondre à la question, parce qu'il existe un foisonnement de travaux sur les indicateurs, qu'on peut résumer en trois catégories :

- la première comprend les indicateurs dont la procédure d'élaboration et la méthode de calcul sont opaques et relèvent du bricolage : il n'y a pas grand chose à en tirer ;

- la seconde repose sur une modélisation globale, adaptée à l'examen de la politique publique d'un pays. L'exemple le plus connu est le modèle " Pression-Etat-Réponse " mis au point par l'OCDE [2] pour évaluer les performances environnementales des pays membres : à l'aide de ce modèle, l'OCDE donne des lignes directrices que ses Etats-membres appliquent chacun à sa convenance. Ce modèle convient à une échelle intergouvernementale, malgré les distorsions d'emploi d'un pays à l'autre. Il est utilisé par l'IFEN [3] qui compte l'adapter aux régions, départements et bassins-versants français ;

- la troisième consiste à modéliser le jeu des différents ensembles impliqués dans le problème posé : c'est la voie choisie dans le présent article $[4,5,6]$. Après avoir exposé une méthode de construction des indicateurs, proposée par l'AFNOR, on s'efforce de situer l'agence de l'eau dans l'ensemble des systèmes agissant sur le régime des eaux, d'analyser leurs influences et interactions, avant de revenir aux indicateurs proprement dits.

\section{I $\square$ LA MÉTHODE DE CONSTRUCTION DES INDICATEURS ET DES TABLEAUX DE BORD}

Il paraît indispensable que la démarche de construction des indicateurs et des tableaux de bord repose sur une méthode éprouvée ; celle d'O. Cérutti et de B. Gattino [7] est résumée ci-après en déroulant les sept étapes suivantes et en les transposant a priori au problème posé : à ce stade, il s'agit davantage d'exemples possibles, imaginés pour illustrer la méthode et écrits en style télégraphique comme dans la plupart des rapports de ce type. 
1 - Identification du champ : mesurer l'efficacité de l'action financière des agences de l'eau sur le milieu et la santé publique.

2 - Les objectifs poursuivis. Comme chaque agence dispose de trois leviers pour agir sur le milieu :

- les redevances,

- les primes à la dépollution,

- les aides à la réalisation d'ouvrages,

il convient de mettre en lumière les liens entre ceux-ci sachant qu'ils ne peuvent être dissociés dans l'évaluation de l'action financière du "système agence", lequel ne se résume pas à leur addition.

3 - Les variables retenues. Chaque levier a son caractère incitatif propre :

- celui des redevances incite à moins prélever et à moins polluer,

- celui des primes vise à obtenir un bon fonctionnement des dispositifs d'épuration,

- celui des aides permet de répondre à la demande des maîtres d'ouvrage, soumis eux-mêmes à une demande sociale, voire économique, et aux contraintes réglementaires.

\section{4 - Les paramètres correspondant aux variables} retenues :

- efficacité de la gestion financière,

- caractéristiques de l'ouvrage,

- entrées et sorties de l'ouvrage,

- amont et aval de l'impact sur le milieu,

- satisfaction des riverains et des usagers des milieux aquatiques,

- satisfaction des maîtres d'ouvrage,

- respect des contraintes législatives et réglementaires.

Même si cette liste paraît déjà longue, elle est loin d'être complète. Par exemple on peut ajouter :

- efficacité économique de la gestion financière, notamment en création d'emplois,

- impact des nouveaux emplois sur le milieu récepteur.

\section{5 - Les indicateurs}

Ils sont de deux natures :

- indicateurs de satisfaction à établir sur la base d'enquêtes périodiques,

- indicateurs de performance : chaque indicateur fait l'objet d'une collecte permanente et spécifique de données élémentaires, de manière à voir les évolutions. Compte tenu de ce qui précède, et si l'on ne prend pas la précaution de se limiter aux données de base produites de façon régulière par les services opérationnels qui s'en servent dans leur travail quotidien, on entreprend la construction d'un système

\section{Le modèle de l'OCDE}

Le modèle OCDE s'applique à l'environnement en général. Lorsqu'on le décline dans le domaine de l'eau, la signification des trois termes est la suivante :

- PRESSION se traduit directement par les prélèvements et par les rejets et indirectement par tout changement affectant le régime des eaux, par exemple le changement d'occupation des sols, la réduction d'un champ d'inondation, etc.

- ETAT se rapporte aux milieux aquatiques mais aussi à l'état sanitaire des populations,

- REPONSE traduit d'abord l'action du pays faisant l'objet d'une évaluation de ses performances environnementales pour réduire la PRESSION ou ses effets sur le milieu ou la santé publique : à ce titre la création des agences de l'eau, la traduction des directives communautaires dans le droit français sont des réponses institutionnelles, la création de stations d'épuration et leur fonctionnement sont des réponses opérationnelles.

d'information déconnecté des préoccupations des acteurs impliqués et très vite impossible à mettre à jour et à faire fonctionner [8].

\section{6 - La construction du tableau de bord.}

En accord avec l'utilisateur final, on procède au choix d'un nombre réduit d'indicateurs qui constituent son tableau de bord personnalisé. L'idée poursuivie est que d'un coup d'œil, ce responsable puisse voir l'essentiel des variations de ces indicateurs, avec une signalisation particulière des évolutions préoccupantes. Chaque jour ou chaque semaine, les données de base font l'objet de traitements divers d'élaboration et de calculs statistiques, pour mettre à jour l'information contenue dans le tableau de bord. Pour rester pérenne, ce travail essentiel de mise à jour doit être profondément ancré dans l'activité opérationnelle de tous les contributaires.

\section{7 - L'optimisation du tableau de bord.}

A ce propos, citons J. Mélèse : "La forme, la nature, la fréquence, la précision des informations dépendent pour beaucoup des hommes qui auront à les utiliser. Une telle considération justifie, par exemple, le fait qu'on ne puisse établir des tableaux de bord standards pour des fonctions, même bien définies, car un tableau de bord est un ensemble d'informations destiné à faire réagir un responsable de manière à améliorer sa maîtrise sur les phénomènes ; il doit donc être adapté à la personnalité du responsable et au style de gestion de l'entreprise" ([9] p. 46).

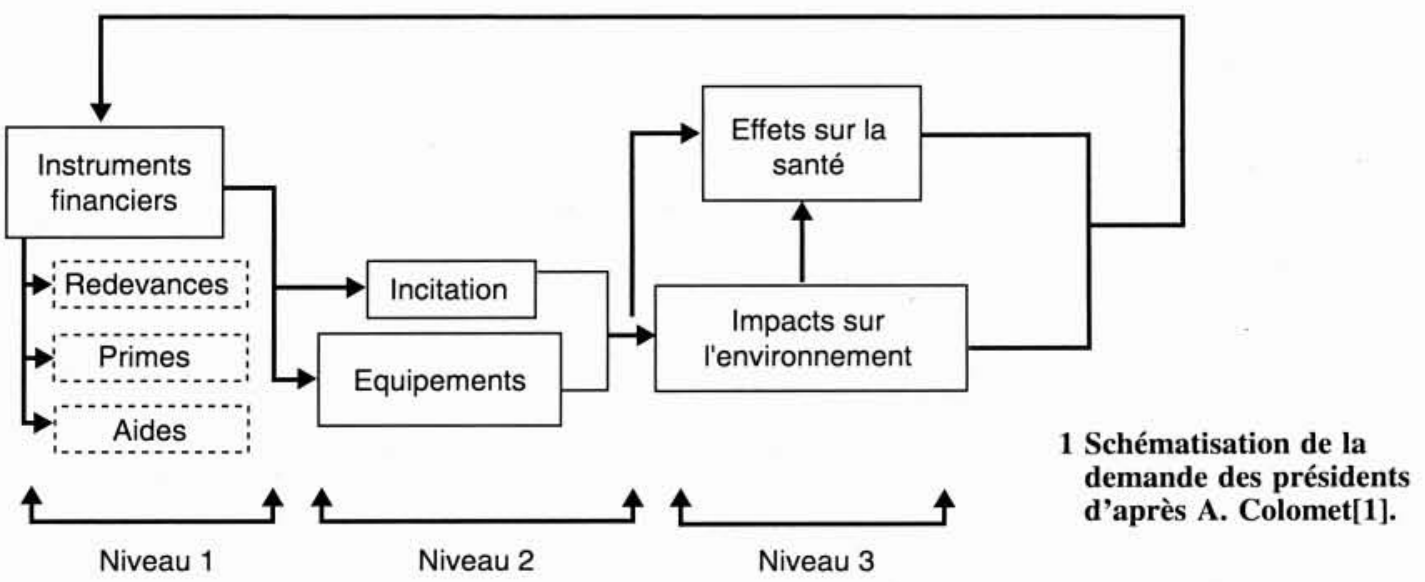




\section{II — UN CONTEXTE DE SYSTÈMES COMPLEXES}

Cette remarque de J. Mélèse signifie qu'un tableau de bord s'applique non seulement à une entreprise et une seule, mais surtout à un type d'acteurs, voire à un seul acteur. Le problème concernant l'évaluation de l'action financière de l'agence devient alors très difficile à exposer et à résoudre, parce qu'il met en jeu différents ensembles complexes et leurs interrelations : le fait est que les acteurs représentant certains de ces systèmes auront chacun une finalité différente, donc une vue différente sur le contenu et sur les moyens de l'évaluation.

Enumérons ces ensembles disjoints, en les classant en trois catégories : "naturel", "hydraulique finalisé" et "artificiel finalisé", sans oublier celui constitué par l'homme, individu consommateur et usager mais aussi objet, pour ne pas dire indicateur, de santé publique.

\section{1 - Les systèmes naturels :}

- le climat qui traduit les variations locales des états de l'atmosphère, laquelle constitue le moteur du cycle hydrologique avec en entrée l'évaporation et en sortie les précipitations atmosphériques,

- le bassin-versant dont la surface varie avec le point considéré du milieu aquatique récepteur,

- le milieu aquatique, que l'on peut connaitre par des stations d'observation et de mesurage. Dans le cas d'un cours d'eau, ces stations sont situées à l'amont et à l'aval du rejet, chacune correspondant à l'exutoire d'un bassin-versant, l'amont étant gigogne de l'aval.

\section{2 - Les systèmes hydrauliques finalisés :}

- celui de la collecte, du traitement et du rejet d'eaux usées, autrement dit le système d'assainissement, lui-même soussystème d'un site industriel ou d'une collectivité territoriale, avec un responsable : le maître d'ouvrage,

- ceux qui agissent directement sur le milieu par des prélèvements à des fins d'alimentation en eau potable, de refroidissement, d'irrigation, de navigation, de stockage pour soutien d'étiage, de transfert vers un autre bassin... Une partie de ces eaux se retrouve après utilisation, dans les réseaux de collecte cités plus haut..

\section{3 - Les systèmes artificiels finalisés :}

celui des redevances, des primes et des aides, dénommé "agence".

La figure $\mathrm{n}^{\circ} 2$ schématise les flux financiers entre l'agence de l'eau et la collectivité locale, elle-même alimentée par l'usager consommateur d'eau.

L'encadré décrit un autre type d'action du système " agence" concernant la pollution industrielle.

- ceux de la surveillance des milieux naturels, des rejets et des prélèvements, avec des finalités multiples de polices des eaux et de la pêche, de surveillance d'installations classées, de surveillance de l'état sanitaire, de définition d'objectifs de qualité,... le tout dénommé "Etat", sans omettre leurs contraintes financières vérifiées par le ministère du Budget, lequel exerce par ailleurs sa tutelle sur les agences de l'eau, - ceux des "Département" et "Région" qui peuvent interférer avec les précédents, soit pour attribuer des aides à des maîtres d'ouvrage (collectivités), soit pour effectuer des mesures sur les ouvrages et sur le milieu,

- celui de l'Institut Français de l'Environnement (IFEN), chargé de réunir et de publier les statistiques sur les différents secteurs de l'environnement, notamment celui de l'eau, en liaison avec l'agence européenne de l'environnement. Ne serait-ce pas cet organisme qui devrait être chargé de rassembler les données de l'évaluation de l'efficacité de l'action financière des agences de l'eau sur le milieu naturel et la santé publique ?

Pour le moment et pour ne pas complexifier davantage, les systèmes associatifs de protection de la nature, les systèmes de connaissance des chercheurs, les systèmes d'éducation, les systèmes médiatiques et les systèmes du monde agricole sont laissés de côté.

Des trois types de systèmes qui viennent d'être passés en revue - le système naturel, le système hydraulique finalisé, le système artificiel finalisé-, les deux derniers relèvent de la catégorie " finalisé " dont on retiendra la définition qu'en donne J .Mélèse : “Des finalités et des buts étant exprimés sur un environnement, un système finalisé est un ensemble organisé de moyens, méthodes, règles et procédures qui permet d'obtenir des réponses satisfaisantes de l'environnement " ([9] p.53). La distinction introduite entre " hydraulique" (construction physiquement présente sur le

\section{QUELQUES DÉFINITIONS}

Equivalent-habitant : ensemble de grandeurs caractéristiques utilisées pour rendre approximativement comparables les émissions de pollutions d'origines différentes et tirées des textes réglementaires pour le calcul des redevances et des primes de dépollution. L'arrêté du 6 novembre 1996 fixe "la quantité de pollution journalière à prendre en compte pour chaque habitant, pour une durée maximale de 5 ans à compter du ler janvier 1997, ainsi qu'il suit : 90 grammes de matières en suspension ; 57 grammes de matières oxydables ; 0,2 équitox de matières inhibitrices ; 15 grammes d'azote réduit ; 4 grammes de phosphore total ; 0,05 gramme de composés organohalogénés adsorbables sur charbons actifs ; 0,23 métox ". La définition de ces paramètres est donnée dans d'autres textes réglementaires par référence aux normes AFNOR et ISO. Par ailleurs un " tableau des coefficients spécifiques de pollution pour l'estimation forfaitaire " fixe les grandeurs caractéristiques de chaque activité polluante pour les paramètres précités : il existe donc des moyens artificiels d'évaluer et de comparer les pollutions d'origines différentes, sur la base de l'équivalent-habitant.
Indicateur : écart de la valeur d'un paramètre -et son évolution dans le temps- par rapport à une valeur de référence ou à un objectif, exprimé de façon relative ou absolue.

Maître d'œuvre : personne physique ou morale (architecte, bureau d'études, service technique de l'Etat, etc.) responsable de la conception, de la direction et de la surveillance des travaux de construction ainsi que de la vérification des comptes de chantier.

Maître d'ouvrage : personne physique ou morale pour le compte de laquelle est réalisée une construction et qui supporte la responsabilité totale de l'opération.

Paramètre : grandeur ou propriété, mesurable ou estimée, d'un objet, d'un flux ou d'un événement.

Tableau de bord : ensemble organisé de façon compréhensible et lisible pour la prise de décision et présentant des indicateurs régulièrement mis à jour de manière à constater des évolutions et à établir des comparaisons. 
terrain) et " artificiel " (construction institutionnelle à caractère financier ou réglementaire ou statistique) n'affecte pas cette définition.

"A l'opposé ", toujours d'après Mélèse, " on ne peut trouver pour un système naturel une volonté ou une pensée finalisatrice qui lui fixe des objectifs, sauf à tomber dans des interprétations anthropomorphiques ou théologiques (l'univers comme un système artificiel finalisé par Dieu) " ([9], p.63).

De tout cela on peut avancer l'idée que l'indicateur peut avoir plusieurs statuts liés aux types de systèmes sur lesquels il est bâti ou à leurs combinaisons dont la liste peut s'allonger avec la prise en compte des systèmes laissés de côté..

Enfin, quel que soit le type, tous les systèmes rencontrés ici relèvent de la complexité : " la complexité, caractère fondamental, apparaît comme l'incapacité de décrire tout le système et de déduire son comportement à partir de la connaissance du comportement de ses parties " ([9], p.51).

\section{III —ANALYSE STRUCTURELLE DES INFLUENCES ENTRE SYSTÈMES}

La recherche consiste à décrire les interrelations entre les différents systèmes précités et à les comprendre afin d'établir l'impact des ouvrages aidés sur le milieu naturel et la santé publique. Si l'on se limite aux seuls ouvrages aidés, il faudra être capable de dissocier l'effet dissuasif des redevances, pour bien évaluer ce qui relève des aides et des primes, donc de l'optimum financier. Aucun des acteurs maître d'ouvrage, agence de l'eau et autres financiers, maître d'œuvre,... - ne recherche l'optimum technique, puisque la décision finale de réaliser un ouvrage est essentiellement financière sur une base d'arguments techniques plus ou moins fondés. La recherche de l'optimum technique prenant en compte l'impact sur le milieu récepteur est susceptible de générer des études sans fin, de retarder l'action en la rendant incertaine et de nourrir des controverses alors que l'optimum financier est cerné après un tour de table.

1 - La construction du tableau des influences des différents systèmes entre eux : les systèmes choisis et soumis à l'analyse structurelle sont indiqués en légende du tableau.

Le résultat du tableau d'analyse structurelle indiquant l'influence des facteurs énumérés en colonnes A à L, sur ceux (les mêmes) figurant lignes $\mathrm{A}$ à $\mathrm{L}$, est reporté sur un graphe. L'interprétation de ce dernier ne peut être effectuée que par celui qui a distribué les " $\mathrm{x}$ " dans les cellules du tableau, en leur faisant supporter un contenu particulier : il ne faut jamais oublier que la méthode est subjective et que nous nous mouvons dans les sciences de l'imprécis et de l'incertain [11]. A ce stade, il est inutile d'évaluer le degré de l'influence en notant chaque cellule de 0 à 3 " $\mathrm{x}$ ", comme le proposent Probst et Ulrich [12].

\section{2 - Commentaires sur les influences :}

a) le climat a un rôle primordial sur la visibilité de l'impact de l'ouvrage, variable selon qu'il y a crue ou étiage (voir encadré sur le taux de collecte à Achères) ;

b) le bassin-versant a lui aussi un rôle : sa surface influence directement l'importance du cours d'eau ; par ailleurs, le changement d'occupation des sols peut entraîner un bruit de fond supplémentaire, voire même un changement de régime ;

c) le maître d'ouvrage à qui échoit la prise en charge de l'ouvrage, doit maintenant surveiller son fonctionnement (autocontrôle) et dans certains cas mesurer son impact sur le milieu aquatique à l'aide de stations situées à l'amont et à l'aval du rejet (ou de la prise d'eau si c'est un ouvrage de prélèvement) ;

d) le système "agence" n'intervient que sur l'estimation des redevances et des primes et sur l'attribution des aides : il

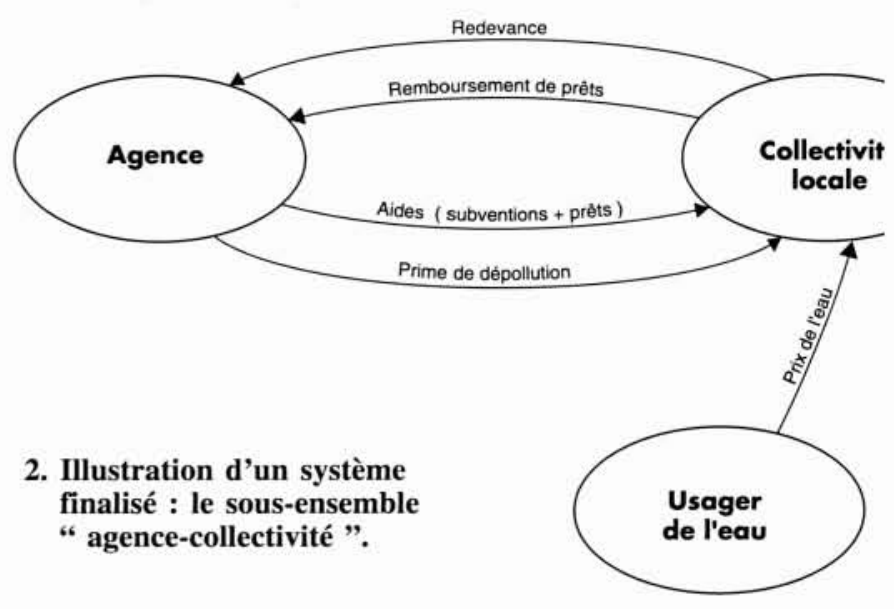

\section{L'action du système " agence" sur la pollution industrielle.}

Dans le cas de la pollution industrielle, l'agence : calcule des redevances brutes, soit forfaitairement selon les textes réglementaires, soit sur la base de la mesure des rejets après dénonciation du forfait par l'une des parties. (il existe aussi une redevance "prélèvement ");

- calcule la prime correspondant à la pollution éliminée par le dispositif d'épuration mis en place par l'industriel ; - perçoit la redevance nette, différence des deux termes précédents ;

- aide l'industriel qui veut dépolluer plus, soit à la source (technologie propre), soit au niveau du rejet, en lui attribuant un prêt ou une subvention pour des travaux réalisés.

Dans ce cas on voit bien que l'agence dissuade l'industriel de polluer davantage, l'incite à réaliser des travaux de dépollution par le jeu des redevances, des primes et des aides : la situation se stabilise lorsque l'industriel ne trouve plus d'intérêt à la réalisation de nouveaux travaux. La course à une amélioration de la dépollution ne reprend que lorsqu'un élément nouveau est introduit dans le système : cas d'un changement de production ou d'une nouvelle redevance. Cet exemple montre qu'un système artificiel (le système financier des agences de l'eau), complètement déconnecté du milieu naturel, est censé avoir une influence sur ce dernier, tout en ignorant à la fois son état initial, supposé représenté par celui de l'amont du rejet, et ses états actuel et ultérieur. Autrement dit, le système d'observation de la qualité des cours d'eau ayant pour finalité d'évaluer l'impact des ouvrages financés n'existe pas dans l'actuel système d'information de l'agence.

n'a pas vocation à s'occuper du milieu récepteur ni du milieu naturel ; s'il le fait, c'est uniquement parce que ses instances de décision l'y autorisent dans des limites très faibles, sans contrepartie financière (absence de redevances ad hoc) ;

e) c'est à l'Etat que les textes législatifs et réglementaires attribuent l'autorisation, la surveillance et le contrôle des rejets et des prélèvements et de leur impact sur le milieu récepteur par rapport aux objectifs de qualité et aux risques sanitaires ;

f) en revanche, les textes confiant à l'Etat l'inventaire du degré de pollution des cours d'eau ont été abrogés avec la dernière loi sur l'eau sans textes de substitution : il y a un vide juridique qui n'empêche ni l'Etat ni l'agence, à laquelle l'Etat demande des fonds de concours, de participer au 
Tableau des influences des différents systèmes entre eux ([10], notamment p. 122 sqq "Du bon usage de l'analyse structurelle").

\begin{tabular}{|c|c|c|c|c|c|c|c|c|c|c|c|c|c|}
\hline $\begin{array}{c}\text { de } \\
\text { sur }\end{array}$ & A & B & C & D & E & F & G & H & I & J & K & L & Total \\
\hline A & & $\mathrm{x}$ & & & & & & & & & & & 1 \\
\hline B & $\mathrm{x}$ & & $\mathrm{x}$ & $\mathrm{x}$ & $\mathrm{x}$ & & & & $\mathrm{x}$ & $\mathrm{x}$ & & $\mathrm{x}$ & 7 \\
\hline C & $\mathrm{x}$ & $\mathrm{x}$ & & & & $\mathrm{x}$ & $\mathrm{x}$ & $\mathrm{x}$ & $\mathrm{x}$ & $\mathrm{x}$ & & $\mathrm{x}$ & 8 \\
\hline D & $\mathrm{x}$ & $\mathrm{x}$ & & & & $\mathrm{x}$ & & $\mathrm{x}$ & $\mathrm{x}$ & $\mathrm{x}$ & & $\mathrm{x}$ & 7 \\
\hline E & $\mathrm{x}$ & $\mathrm{x}$ & & & & & $\mathrm{x}$ & $\mathrm{x}$ & $\mathrm{x}$ & $\mathrm{x}$ & & $\mathrm{x}$ & 7 \\
\hline F & $\mathrm{x}$ & $\mathrm{x}$ & & $\mathrm{x}$ & & & & $\mathrm{x}$ & $\mathrm{x}$ & $\mathrm{x}$ & & $\mathrm{x}$ & 7 \\
\hline G & $\mathrm{x}$ & $\mathrm{x}$ & $\mathrm{x}$ & $\mathrm{x}$ & $\mathrm{x}$ & $\mathrm{x}$ & & $\mathrm{x}$ & $\mathrm{x}$ & $\mathrm{x}$ & & $\mathrm{x}$ & 10 \\
\hline H & & & $\mathrm{x}$ & $\mathrm{x}$ & $\mathrm{x}$ & & & & $\mathrm{x}$ & $\mathrm{x}$ & & $\mathrm{x}$ & 6 \\
\hline $\mathrm{I}$ & $\mathrm{x}$ & & & & & $\mathrm{x}$ & $\mathrm{x}$ & $\mathrm{x}$ & & & $\mathrm{x}$ & $\mathrm{x}$ & 6 \\
\hline J & $\mathrm{x}$ & & $\mathrm{x}$ & $\mathrm{x}$ & $\mathrm{x}$ & & & $\mathrm{x}$ & $\mathrm{x}$ & & & $\mathrm{x}$ & 7 \\
\hline K & $\mathrm{x}$ & $\mathrm{x}$ & & & & $\mathrm{x}$ & $\mathrm{x}$ & $\mathrm{x}$ & $\mathrm{x}$ & $\mathrm{x}$ & & $\mathrm{x}$ & 8 \\
\hline L & $\mathrm{x}$ & $\mathrm{x}$ & $\mathrm{x}$ & $\mathrm{x}$ & $\mathrm{x}$ & $\mathrm{x}$ & $\mathrm{x}$ & $\mathrm{x}$ & $\mathrm{x}$ & $\mathrm{x}$ & $\mathrm{x}$ & & 11 \\
\hline Total & $\mathrm{10}$ & 8 & 5 & 6 & 5 & 6 & 5 & 9 & 10 & 9 & 2 & 10 & \\
\hline
\end{tabular}

A : climat, principalement les éléments qui font les régimes des cours d'eau et leur variabilité.

B : bassin-versant, principalement sa surface et ses changements d'occupation.

C : maître d'ouvrage ou exploitant, responsable de l'ouvrage dont l'impact est évalué.

D : maîtres d'ouvrage situés à l'amont de l'ouvrage considéré et désignés ici "ouvrages amont".

E : maîtres d'ouvrage situés à l'aval de l'ouvrage considéré et désignés ici "ouvrages aval".

F : cours d'eau à l'amont de l'ouvrage considéré, équipé d'une station de mesure.

$\mathrm{G}$ : cours d'eau à l'aval de l'ouvrage considéré, équipé d'une station de mesure.

$\mathrm{H}$ : système agence (redevances, primes et aides).

I : système Etat (surveillance des installations classées, police des eaux, surveillance sanitaire, aménagement du territoire,...).

$\mathrm{J}$ : système Département et Région (SATESE ${ }^{1}$, aides financières, observatoires environnementaux).

$\mathrm{K}$ : institut français de l'environnement (IFEN).

L : les consommateurs et les usagers, eux-mêmes indicateurs de l'état de la santé publique, et leurs associations.

maintien des réseaux de mesure en général et du réseau national de bassin en particulier ; ce dernier n'est cependant pas finalisé pour observer l'efficacité de l'action financière de l'agence ;

g) Les consommateurs et les usagers sont ceux qui payent directement ou indirectement. En outre,

- ils participent à l'élection du parlement (démocratie représentative), lequel établit les lois qui vont être transformées en décrets, arrêtés et circulaires d'application par les services de l'Etat ;

- ils élisent aussi leurs représentants locaux qui vont constituer les maîtres d'ouvrage publics ;

- ils agissent par ailleurs au sein de leurs associations (démocratie participative) dont la voix est écoutée avec attention dans les instances de l'agence (comité de bassin, conseil d'administration) où l'Etat est minoritaire ${ }^{2}$;

- enfin, ils traduisent l'état de la santé publique.

\section{3 - Commentaires sur la figure $n^{\circ} 3$ résultant du tableau.}

Chaque système de $\mathrm{A}$ à $\mathrm{L}$ est figuré par un point avec en ordonnée le total de son influence (colonne) et en abscisse le total de sa dépendance (ligne).

La figure $\mathrm{n}^{\circ} 3$ distingue bien les différents systèmes : on note que les systèmes naturels s'échelonnent de l'indépendance quasi totale (climat) à une grande dépendance pour l'aval du cours d'eau, nuancée par une possibilité de régulation par rétroaction ${ }^{3}$ sur les systèmes artificiels (réglementaires et financiers), nuances que l'on retrouve pour les

(1) Service d'Assistance Technique à l'Exploitation des Stations d'Epuration. (2) C'est la chance des agences de l'eau, comparée à la situation des autres établissements publics à caractère administratif, où l'Etat est omnipotent.

(3) Faute de place, les rétroactions ne sont pas représentées sur la figure $n^{\circ} 3$. systèmes physiques. Le système artificiel représenté par l'IFEN se trouve dans un état de totale dépendance et, pour le moment, sans grande possibilité de rétroaction. Quant à l'Etat, en s'appuyant sur les textes existants et sur ses très nombreux services sectorisés, son influence est très forte, et c'est ce qui ressort du graphique ; or l'Etat n'est pas en mesure de faire appliquer intégralement ses textes réglementaires et d'en contrôler les effets. Par exemple, l'un des objectifs principaux de la loi sur l'eau du 3 janvier 1992 a été de remettre en selle la police des eaux qui fut défaillante malgré la loi du 16 décembre 1964 et ses très (trop ?) nombreux textes d'application. Pour le moment, la police des eaux reste diluée dans un certain nombre d'organismes rattachés à différents ministères (Agriculture, Transport, Equipement, Industrie) dont ce n'est pas le premier souci : en tout cas, ce souci viendra toujours après celui plus gratifiant de maitre d'œuvre. Le personnel de la police des eaux est en principe mis à la disposition du ministère de l'Environnement, mais il reste très insuffisant. Par ailleurs, sa situation le met en présence de deux patrons, un hiérarchique (son ministère d'origine) qui le paye et un fonctionnel (le ministère de l'environnement) : elle correspond à celle de l'adage "qui a deux patrons, n'en a pas" ! Enfin les arrêtés préfectoraux d'autorisation de prélèvement ou de rejet stipulent souvent des dispositifs de mesures à la charge du maître d'ouvrage : les données qui en résultent ne sont pas aisément accessibles ni facilement exploitables ; il y a pourtant là un gisement potentiel important de données nécessaires à une évaluation de l'impact des ouvrages et, par extension théorique, de l'action financière des agences de l'eau sur le milieu.

Dans le raisonnement précédent, nous avons pris la précaution de distinguer l'ouvrage étudié de ceux nombreux situés soit à l'amont soit à l'aval, en supposant que des mesures différentielles sur le milieu récepteur à l'amont et à 
l'aval, conjuguées avec celles effectuées sur l'ouvrage luimême, devraient permettre d'évaluer et de qualifier leurs influences respectives.

Enfin le consommateur et l'usager prennent une place particulière dans la modélisation et figurent les enjeux principaux de la politique de l'eau. De fait l'homme est bien l'enjeu essentiel parce qu'il agit sur tous ces systèmes, excepté le climat ${ }^{4}$, et subit les rétroactions de tous : ainsi, quelles que soient les précautions prises initialement, l'homme se situe, par construction, au centre des préoccupations, que ce soit son environnement -en l'occurrence les milieux aquatiques- ou sa santé. C'est à méditer.

\section{IV — ESSAI DE DESCRIPTION DES SYSTÈMES ET DE LEURS RELATIONS}

Une autre façon de modéliser les mêmes systèmes complexes est proposée avec la figure 4 : dans cette représentation, on s'attache à identifier ce qu'il faut connaître pour pouvoir formaliser le (ou les) problème(s) issu(s) de la question initiale. On y retrouve globalement les mêmes systèmes avec les principaux flux échangés entre eux : ces flux peuvent être financiers, informatifs, hydriques naturels ou hydriques artificialisés. Or c'est de l'examen de l'état des systèmes et du contenu de ces flux, lesquels traduisent ce que font les systèmes, qu'il sera possible, ou non, de tirer des variables et les indicateurs correspondants. Rappelons qu'il s'agit de voir plus clair dans le passage du financement à l'état du milieu ou à celui de la santé publique. C'est cette préoccupation qui sous-tend les commentaires suivants sur les entités (les ronds et les ovales) et sur les flux (les flèches qui les relient).

\section{1 - Les flux financiers entre l'agence et le maître \\ d'ouvrage peuvent être de trois natures :}

- les redevances sont établies sur la base d'une information annuelle (déclaration ou mesure) concernant l'activité de l'ouvrage et/ou de l'usage assujetti : le montant financier peut dans certains cas être directement relié à une réalité physique de l'eau, par exemple " volumes prélevés " ou " pollution brute ", ou indirectement, par exemple " surfaces irriguées "ou "habitant-équivalent" ;

- les primes à la dépollution nécessitent annuellement des informations sur le fonctionnement d'une seule catégorie d'ouvrages : les dispositifs d'épuration ;

- les aides attribuées pour des travaux à réaliser par le maître d'ouvrage posent le problème du coût de l'ouvrage : à l'expérience, chaque ouvrage est un cas particulier et pour un même niveau de performance attendu, il y a tellement de différences sur les contraintes de toutes natures affectant chaque site qu'on n'obtient pas de relation suffisamment significative entre la taille de l'ouvrage et son coût. Les notions de " juste prix ", de " prix de référence " ou de " prix-plafond" sont pratiques pour l'instructeur du dossier d'aide ; cependant elles sont encore directement reliées au seul investissement. De nombreux obstacles restent à surmonter pour intégrer dans ces prix la durée de vie probable de l'ouvrage, son coût de fonctionnement et sa fiabilité (nombre de défaillances et leurs impacts sur le milieu ou la santé publique)

\section{2 - Un nombre important de types d'ouvrages est concerné} par le programme d'intervention de l'agence.

Pour chaque type d'ouvrage, il est très important de trouver les caractéristiques qui traduisent le mieux le lien entre le financement et ce pourquoi l'ouvrage est réalisé. Par

(4) Nous laissons volontairement de côté tout le débat sur le changement climatique lié à l'activité humaine, laquelle dépasse très largement le cadre des seules activités sur l'eau, objet du présent article. exemple, dans le cas d'un réseau d'assainissement, le nombre d'habitants-équivalents raccordés semble une donnée plus significative que la longueur, le diamètre et le matériau des tuyaux qui le composent et qui pourtant expliquent mieux une partie de son coût. L'agence doit avant tout garder la trace des caractéristiques contractuelles de l'attribution de son aide ; le recueil éventuel des données concernant une influence sur le milieu ou la santé publique passe après.

\section{3 - Les maîtres d'ouvrage recouvrent plusieurs catégories}

fort différentes, elles-mêmes très contrastées :

- les collectivités territoriales dotées de budgets annuels "eau et assainissement" variant de plusieurs milliards (syndicat interdépartemental d'assainissement de l'agglomération parisienne) à quelques dizaines de milliers de francs (syndicat d'une zone rurale) :

- les industriels et leurs différentes branches d'activité qui ont un impact variable sur le milieu ;

- les agriculteurs et leur diversité (éleveurs, céréaliers, viticulteurs, betteraviers, maraîchers,... ).

\section{4 - Les textes législatifs et réglementaires ont une} influence sur le dimensionnement de l'ouvrage

Par exemple, les textes actuels issus de la directive européenne sur les eaux résiduaires urbaines tirent vers le haut les niveaux de collecte et de traitement des ouvrages d'épu-

\section{Pourquoi le taux de collecte de la station d'Achères chute-t-il de 9 points entre 1993 et 1994 ?}

Le taux de collecte, exprimé en \%, est le rapport de la pollution entrant en station d'épuration, à la pollution produite sur la zone de collecte pour épuration et raccordée à la station. Ce calcul peut se faire sur différents paramètres : généralement on choisit les matières oxydables, avec au numérateur le résultat de la mesure à l'entrée de la station d'épuration et au dénominateur une évaluation théorique basée sur 57 grammes de matières oxydables de pollution émise par jour et par habitant, ou " équivalenthabitant".

La comparaison des résultats obtenus en 1993 et en 1994 montre une chute de $9 \%$. C'est énorme, et pourtant c'est normal : en effet la zone de collecte d'Achères est constituée de réseaux unitaires qui véhiculent l'essentiel des eaux pluviales, lesquelles se mélangent aux eaux usées et provoquent une dilution des concentrations de matières oxydables. Comme le débit entrant à la station d'Achères est limité à une trentaine de mètres cubes par seconde, le surplus est évacué directement en Seine soit par surverses des déversoirs d'orage au fil des réseaux unitaires, soit par un by-pass qui détourne le débit excédentaire juste avant la station d'épuration. Or 1994 est une année de plus forte hydraulicité que 1993. En 1994 il est tombé à Paris-Montsouris $700 \mathrm{~mm}$ de pluie contre $663 \mathrm{~mm}$ en 1993. On retrouve ici un peu de l'influence du climat, identifiée lors de l'analyse structurelle.

Mais ce n'est pas tout : en 1994 commencent les chantiers d" "Eole " et de " Météor". Pour réaliser les travaux, il faut mettre au sec les ouvrages en construction, en pompant la nappe phréatique et en rejetant l'eau fortement chargée en matières en suspension dans le réseau d'assainissement qui aboutit à Achères : de fait le taux de collecte des matières en suspension augmente de $15 \%$ entre 1993 et 1994, résultat à l'opposé de celui constaté sur les matières oxydables!

Cet exemple montre à la fois le caractère artificiel des calculs, la nécessité de bien appréhender la réalité du terrain et l'insuffisance de connaissance des processus fondamentaux. 
ration, à un point tel que les sommes à investir pour respecter les échéances dépassent les capacités financières de certaines collectivités et qu'il aurait fallu augmenter, encore et plus fortement qu'au VIème programme, les redevances de l'agence de l'eau. En ce qui concerne l'Etat, il reste beaucoup à faire pour détailler les divers types d'actions, potentielles et réelles, ayant ou pouvant avoir un impact sur le milieu et la santé publique ; cette dernière semble cependant bien mieux lotie dans le domaine de l'alimentation en eau potable $[13,14]$.

\section{5- D'un type d'ouvrage à l'autre, l'influence sur le milieu est de nature totalement différente}

Prenons trois exemples :

- celui du prélèvement d'irrigation : la ressource est amputée d'un volume qui a un impact d'autant plus important qu'il y a concomitance des sécheresses pédologique et hydrologique : il s'ensuit des conflits d'usages que l'Etat tente d'empêcher par des arrêtés préfectoraux. Dans ce cas précis, lorsque l'agence finance des forages d'irrigation, elle participe à un impact jugé négatif sur le milieu, d'autant que la quasi totalité de l'eau prélevée s'évapore. A ce propos, il convient de rappeler un passage de l'article 14 de la loi du 16 décembre 1964 relative au régime et à la répartition des eaux et à la lutte contre leur pollution: " l'agence établit et perçoit sur les personnes publiques ou privées des redevances, dans la mesure où ces personnes publiques ou privées rendent nécessaire ou utile l'intervention de l'agence ou dans la mesure où elles y trouvent leur intérêt ". Or il existe une redevance " irrigation " qui autorise l'agriculteur à demander une aide et l'agence à l'accorder. Il est bon de rappeler que la mission première de l'agence n'est pas l'entretien ou la restauration du milieu naturel, même si elle y contribue dans la majorité de ses actions :

- celui de la réalisation d'un barrage-réservoir destiné au soutien d'étiage : l'idée même de ce type d'ouvrage est combattue par des associations de protection de la nature, alors que les besoins d'alimentation en eau potable doivent être satisfaits en toute sécurité. C'est le cas type d'une influence controversée : qui croire en la matière et quel indicateur faut-il mettre en place?

- celui d'un réseau d'assainissement qui va permettre de raccorder quelques centaines d'habitants : le point de vue hygiéniste indiquera une amélioration de la santé publique ; si cette extension de réseau est raccordée à une station d'épuration déjà en surcapacité, la situation du milieu récepteur va être aggravée.

D'où la question : comment va-t-on juger de l'efficacité de l'action de l'agence qui aura aidé les trois projets ? Peuton imaginer un indicateur qui agglomère ces trois affaires ?

\section{6 - La connaissance du milieu naturel et de son évolution est insuffisante.}

- Les rivières font l'objet de mesures physico-chimiques depuis plus de 25 ans avec la création de l'Inventaire National de la Pollution (INP) auquel s'est substitué le Réseau National de Bassin (RNB) en 1987. Une étude récente sur le degré de signification $[15,16]$ de ces mesures a constaté :

- l'absence de finalité clairement identifiée des réseaux actuels dits de "suivi patrimonial ",

- l'insuffisance de la fréquence des prélèvements d'échantillon, du nombre de paramètres,

- l'impossibilité d'effectuer des bilans, en termes de

flux globaux et d'impact d'ouvrage ou de zone de rejets.

Cependant les réseaux actuels peuvent donner une bonne idée de l'évolution des paramètres mesurés depuis 1971.

- Par ailleurs, notre connaissance des matières toxiques piégées dans les sédiments et relarguées vers l'aval au hasard des crues est très fragmentaire, à l'exception de la Seine à l'aval de Poses où ce suivi est réalisé depuis 30 ans. La connaissance hydrobiologique s'appuie sur un indice de biodiversité, l'IQBG (Indice de Qualité Biologique Globale), mais ne répond pas à des questions toutes simples du genre : “ est-ce que le poisson pêché est mangeable et possède sa qualité gustative normale "? Enfin, la connaissance bactériologique du milieu était réduite à quatre paramètres qui ne renseignent pas sur la présence de virus. Tous ces points très difficiles à traiter ont fait, font et feront l'objet d'études approfondies dans le cadre d'études inter-agences et des programmes PIREN [17].

- Enfin, sur quelles références faut-il s'appuyer pour caractériser le milieu à préserver ? P. Balland [18] suggère une approche fonctionnelle des milieux aquatiques de surface, fondée sur la vie piscicole et la biodiversité et propose de prendre au cas par cas une référence tenant compte d'une anthropisation souvent très ancienne.

\section{7 - La connaissance de l'impact sur le milieu récepteur est difficile à établir}

L'essentiel des rares mesures effectuées à ce jour a été fait dans le cadre de la police des eaux, mais n'est pas aisément accessible. Cependant, un volume prélevé, mesuré avec un compteur (système géré par l'agence pour établir ses redevances), se soustrait sans difficulté du volume disponible dans le milieu, à condition que ce dernier soit bien connu, ce qui n'est pas toujours le cas.

Le cas des rejets est beaucoup plus complexe :

- un rejet est mesuré la plupart du temps selon une liste de paramètres, laquelle peut s'avérer insuffisante, notamment dans le domaine des toxiques ; par ailleurs la fréquence des mesures et leur calendrier doivent être établis cas par cas dans un souci de pertinence ;

- les matières rejetées vont soit se diluer (matières dissoutes), soit se transformer (nitrification et dénitrification, par exemple), soit être transportées en suspension, soit se déposer : dans tous les cas, la mesure de leur impact sur le milieu entraîne des questions que l'on pense résoudre par un dispositif de mesures à l'amont et à différents endroits à l'aval, réduit aux seuls paramètres physico-chimiques sur eau brute (se reporter aux textes ${ }^{5}$ récents imposant ce système aux maîtres d'ouvrage publics ou privés). Sans évoquer les rapports de dilution entre le rejet et le milieu qui peuvent rendre impossible la mesure, les aspects " piégeage dans les sédiments" et " hydrobiologie " ne sont pas traités. Il reste encore beaucoup à faire dans ce domaine !

\section{8 - La modélisation proposée, outre son caractère géné-} ral, comporte forcément des lacunes

a) Les maîtres d'œuvre se répartissent entre le secteur privé, les services techniques de certains maîtres d'ouvrage, les services de l'Etat et quelques services départementaux : quelle que soit son origine, le maître d'oeuvre conseille le maître d'ouvrage qui prend seul les décisions importantes et en supporte la responsabilité totale.

b) Il en est de même pour les exploitants, lesquels ont un rôle primordial dans la qualité du fonctionnement des ouvrages.

c) Le consommateur ou l'usager est d'abord préoccupé par l'augmentation du prix de l'eau et il fait vite le lien avec l'augmentation des redevances de la période 1992-1996. L'aspect sanitaire est passé en général sous silence, sauf accident localisé ou cas épisodiques bien connus, comme par exemple la contamination de coquillages : mais dans ce cas l'action de l'agence n'est pas directement mise en cause. Seule une minorité d'usagers met en avant l'insuffisance de l'action de l'agence sur l'environnement et, là, on retrouve le prix de l'eau.

(5) Arrêté du 1 mars 1993. Décret $94-469$ du 3 juin 1994, arrêté du 22 décembre 1994 et leur circulaire d'application du 12 mai 1995 


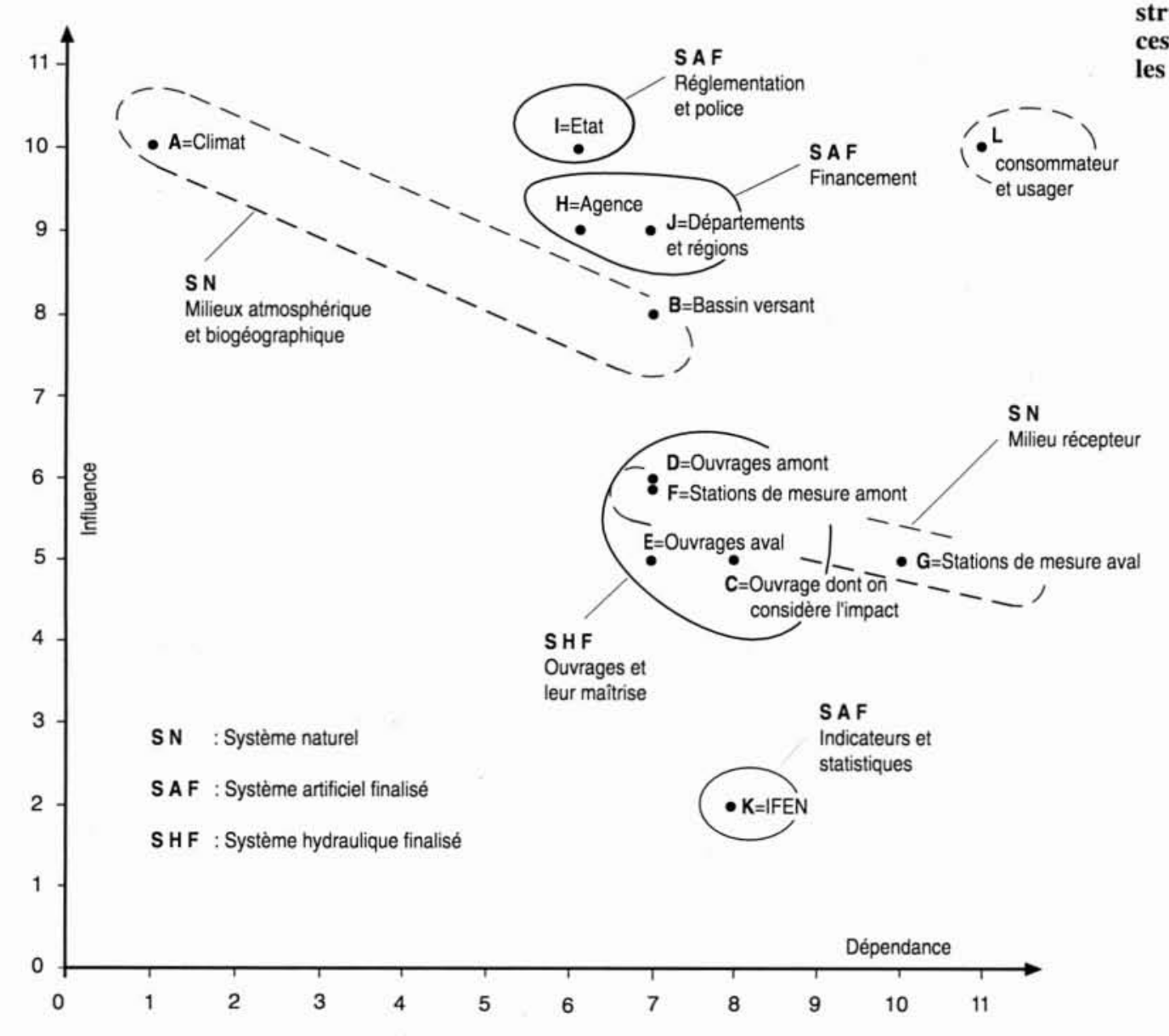

3. Résultat de l'analyse structurelle des influences et dépendances entre les systèmes.

4. Schématisation des différents types de flux entre les systèmes artificiels et naturels.

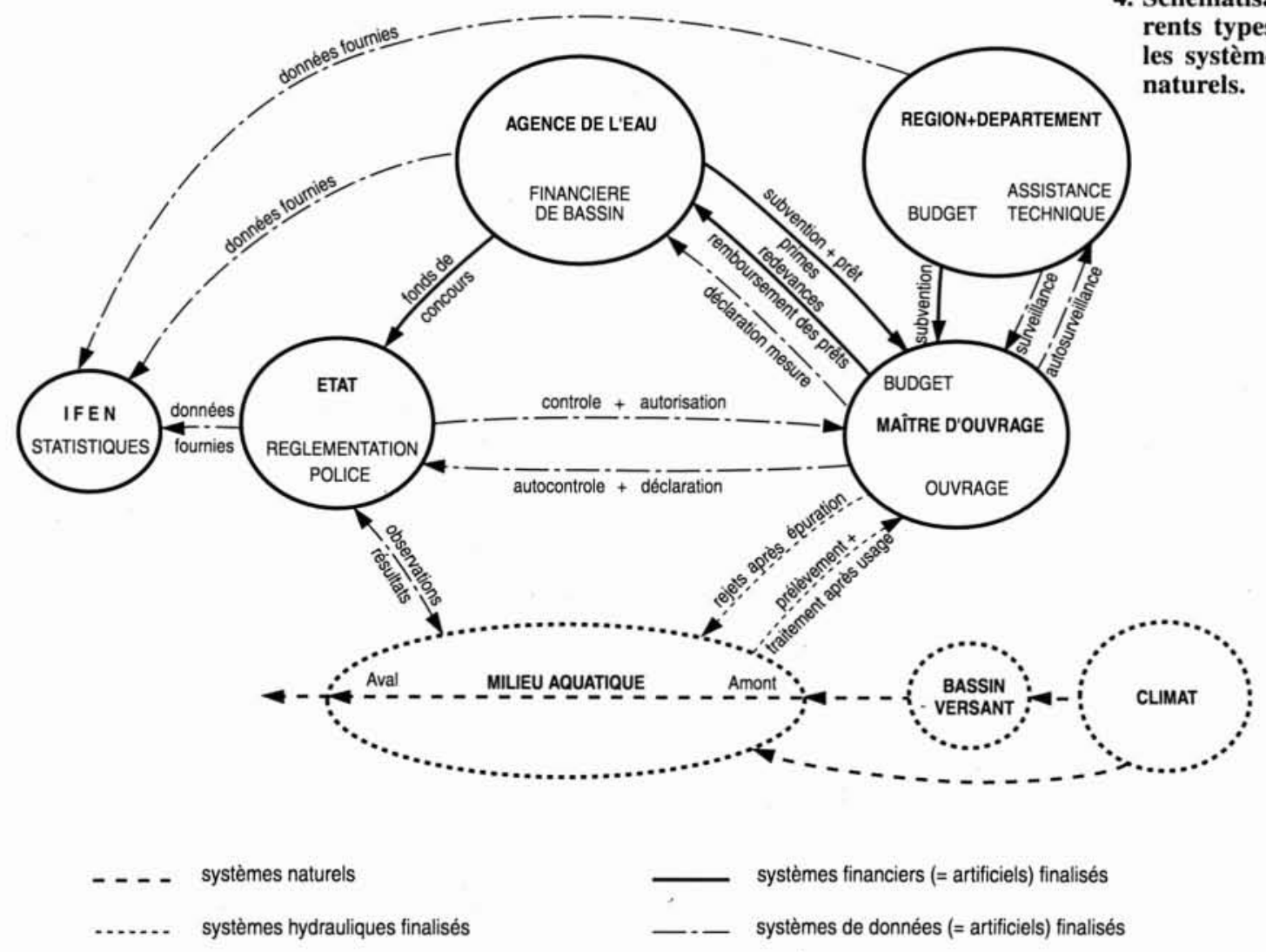


V L'EXISTANT EN MATIÈRE D'INDICATEURS À L'AGENCE DE L'EAU SEINE-NORMANDIE

1 - Les utilisations de données financières répondent à plusieurs finalités visant à :

- établir un planning annuel de référence des recettes, des dépenses et de la trésorerie pour l'année en cours ou à venir, avec mise à jour semestrielle : c'est le référentiel du tableau de bord de gestion,

- gérer l'agence au quotidien : il s'agit de surveiller le niveau de trésorerie qui doit être maintenu entre 0,8 et 1,5 du montant mensuel de l'année budgétaire en cours, en tenant compte des recettes et des dépenses : c'est le tableau de bord nécessaire pour la prise de décision hebdomadaire,

- tenir un tableau de bord trimestriel, rendant compte du déroulement des recettes et des dépenses : pour ces dernières il est fait par ligne-programme (par exemple, les stations d'épuration constituent une ligne-programme), le détail des aides attribuées et celui des paiements effectués au cours du trimestre écoulé, sachant qu'il se passe en moyenne 2,5 ans entre l'attribution d'aides pour travaux à réaliser et le paiement des travaux réellement faits : c'est ce tableau de bord qui permet de proposer des ajustements de réalisation budgétaire en cours d'année.
- rendre compte aux instances de l'agence de l'état de réalisation du programme et du budget de l'année en cours, - renseigner l'annexe du projet de loi de finances consacré aux agences de l'eau et soumis au parlement chaque année.

Ces deux dernières utilisations sont des bilans, plus que des tableaux de bord.

\section{2 - Les indicateurs physiques}

Depuis 1992 et à l'occasion de la mise en œuvre du VIème programme des agences de l'eau, la direction de l'eau du ministère de l'Environnement qui exerce leur tutelle, a défini des indicateurs de suivi dont les résultats doivent être communiqués par chaque agence. L'agence Seine-Normandie n'a jamais donné son accord sur ces indicateurs physiques ; cependant elle fournit annuellement les informations demandées. Le tableau $\mathrm{n}^{\circ} 6$ " indicateurs physiques 1995 " a été publié dans l'annexe à la loi de finances de 1997 (cf copie de la page 67), sans la mention de prudence indiquée dans sa transmission aux ministères de tutelle : "Les données qui figurent dans le tableau ci-dessus, ainsi que les règles observées pour les rassembler sont indiquées avec réserves. Elles seront susceptibles d'être modifiées lorsque des techniques de contrôle auront permis de les valider. Elles constituent donc une première approximation". Dans cet exercice, il

Tableau 6. - Indicateurs physiques 1995. (Extrait de l'annexe à la loi de finances 1997 concernant l'agence de l'eau Seine-Normandie).

\begin{tabular}{|c|c|c|c|}
\hline \multirow{5}{*}{$\begin{array}{l}\text { Assainissement } \\
\text { des } \\
\text { collectivités }\end{array}$} & $\begin{array}{c}\text { Créations de } \\
\text { stations d'épuration } \\
\text { en équivalent-habitant }\end{array}$ & $\begin{array}{l}\text { Capacités totales } \\
\text { Matières en suspension (MES) } \\
\text { Matières oxydables (MO) } \\
\text { Matières azotées (MA) } \\
\text { Matières phosphorées (MP) } \\
\text { Equipements complémentaires }\end{array}$ & $\begin{array}{r}943490 \\
936000 \\
943490 \\
960000 \\
472000 \\
-\end{array}$ \\
\hline & $\begin{array}{l}\text { Améliorations et } \\
\text { extensions de } \\
\text { stations d'épuration } \\
\text { en équivalent-habitant }\end{array}$ & $\begin{array}{c}\text { Capacités totales } \\
\text { MES } \\
\text { MO } \\
\text { MA } \\
\text { MP } \\
\text { Equipements complémentaires }\end{array}$ & $\begin{array}{r}224150 \\
183800 \\
183800 \\
218900 \\
138700 \\
-\end{array}$ \\
\hline & $\begin{array}{c}\text { Extensions des réseaux } \\
\text { de collecte }\end{array}$ & $\begin{array}{l}\text { Capacités créées } \\
\text { en équivalent-habitant }\end{array}$ & 222775 \\
\hline & $\begin{array}{c}\text { Extensions des réseaux } \\
\text { de transport }\end{array}$ & $\begin{array}{l}\text { Capacités créées } \\
\text { en équivalent-habitant }\end{array}$ & 146930 \\
\hline & $\begin{array}{l}\text { Extensions des bassins } \\
\text { de dépollution }\end{array}$ & $\begin{array}{l}\text { Volumes } \\
{\text { en } \mathrm{m}^{3}}\end{array}$ & 370000 \\
\hline \multirow[t]{2}{*}{$\begin{array}{l}\text { Dépollution } \\
\text { des activités } \\
\text { économiques }\end{array}$} & $\begin{array}{l}\text { Quantité supplémentaire } \\
\text { de pollution éliminable } \\
\text { par les industries et } \\
\text { les activités assimilées }\end{array}$ & $\begin{array}{c}\text { MES en } \mathrm{kg} / \text { jour } \\
\text { MO en } \mathrm{kg} / \text { jour } \\
\text { MA en } \mathrm{kg} / \mathrm{jour} \\
\text { MP en } \mathrm{kg} / \mathrm{jour} \\
\text { Matières inhibitrices }(\mathrm{MI}) \text { en Equitox/jour }\end{array}$ & $\begin{array}{r}17576 \\
19562 \\
745 \\
129 \\
705388 \\
\end{array}$ \\
\hline & Elevages & Nombre d'Unités de Gros Bétail (UGB) assainies & 44233 \\
\hline $\begin{array}{l}\text { Gestion de } \\
\text { la ressource }\end{array}$ & $\begin{array}{c}\text { Augmentation de } \\
\text { la capacité des ouvrages } \\
\text { structurants (en millions } \\
\text { de } \mathrm{m}^{3} \text { par an) }\end{array}$ & $\begin{array}{c}\text { Capacités des retenues } \\
\text { d'eau créées } \\
\text { Capacités des ouvrages de transferts } \\
\text { (pour les périodes d'étiage) }\end{array}$ & - \\
\hline \multirow{3}{*}{$\begin{array}{l}\text { Alimentation } \\
\text { en } \\
\text { eau potable }\end{array}$} & $\begin{array}{l}\text { Créations d'unités } \\
\text { de production }\end{array}$ & $\begin{array}{l}\text { Forages en } \mathrm{m}^{3} / \text { jour } \\
\text { Usines de production en } \mathrm{m}^{3} \text { par jour }\end{array}$ & $\begin{array}{r}73560 \\
5960 \\
\end{array}$ \\
\hline & $\begin{array}{l}\text { Interconnexions } \\
\text { et réservoirs }\end{array}$ & $\begin{array}{l}\text { Capacités d'échanges en } \mathrm{m}^{3} / \mathrm{jour} \\
\text { Volumes des réservoirs en } \mathrm{m}^{3 / \text { jour }}\end{array}$ & $\begin{array}{r}164525 \\
-\end{array}$ \\
\hline & $\begin{array}{l}\text { Population supplé- } \\
\text { mentaire (en hab.) } \\
\text { desservie par une eau : }\end{array}$ & $\begin{array}{l}\text { Rendue conforme aux } \\
\text { normes de potabilités } \\
\text { Améliorée ou sécurisée }\end{array}$ & $\begin{array}{r}292880 \\
75953000\end{array}$ \\
\hline
\end{tabular}


s'agit d'indiquer ce que les aides de l'agence vont permettre de réaliser sous forme de travaux, les ouvrages correspondants n'étant opérationnels qu'en moyenne deux ans et demi plus tard. Ce tableau appellerait de très nombreux commentaires et devrait susciter de très nombreuses études pour en critiquer le contenu et l'améliorer. Par exemple, le chiffre de plus de 7 millions d'habitants ayant eu une eau distribuée améliorée ou sécurisée risque de se reproduire chaque année. En effet, les réseaux d'alimentation en eau potable de la mégapole parisienne, à la fois gigantesques et interconnectés, font l'objet d'aides pour travaux tous les ans, ce qui sécurise ou améliore la qualité de l'eau distribuée des Franciliens. En l'état, cet indicateur n'est pas pertinent sur le bassin Seine-Normandie, sans doute parce que l'on mélange plusieurs populations statistiquement distinctes, en particulier celle de la mégapole et celle du monde rural.

Le tableau $\mathrm{n}^{\circ} 7$ " pollutions éliminées en 1994 " présente les résultats de l'application stricte des textes législatifs et réglementaires concernant la pollution éliminée par les ouvrages existants et donnant lieu à l'attribution de primes au bon fonctionnement. Ces données ne sont pas contestables sur le plan administratif et réglementaire. Ici nous sommes dans un système artificiel finalisé de primes à la dépollution qui s'articule sur les systèmes hydrauliques finalisés des stations d'épuration, à l'aide de valeurs théoriques (les " habitants équivalents" collectés), de résultats de mesure de pollution éliminée et de règles de calcul à la fois simples et peu contraignantes : le législateur, dans sa sagesse, a préféré l'efficacité en tablant sur le caractère incitatif d'une prime à la dépollution versée le plus rapidement possible aux maîtres d'ouvrage, plutôt que sur la mesure d'un hypothétique impact sur le milieu récepteur.

En l'état actuel, ce ne sont pas des tableaux de bord : pour le devenir, il faudra établir une valeur de référence propre à chaque rubrique et comparer chaque valeur annuelle à la référence.

Enfin l'appréhension du milieu aquatique et de son évolution pose une série de problèmes qui rendent encore plus difficile la recherche d'indicateurs pertinents d'estimation de l'action financière des agences sur le milieu naturel.

\section{VI $\square$ CONCLUSION}

\section{1 - La réponse correcte à la question posée doit être différée}

En effet, il est possible de construire actuellement les indicateurs suivants :

- gestion financière du budget annuel et du programme quinquennal,

- suivi du fonctionnement des ouvrages avec les données des primes et des redevances,

- suivi de l'évolution des milieux naturels, notamment aquatiques,

et à moyen terme :

- suivi de l'évolution de l'équipement du bassin découlant des décisions d'aides,

- suivi de la satisfaction des usages en relation avec les normes sur les eaux des milieux ${ }^{6}$

Ces indicateurs globaux au niveau du bassin mélangent des populations statistiquement différentes, sur des étendues géographiques trop importantes : pour réduire cet inconvénient, il semblerait judicieux d'effectuer ce travail au niveau de chaque $\mathrm{SAGE}^{7}$ et d'agglomérer les informations obtenues au niveau du bassin. A l'appui de cette suggestion, il convient de rappeler que la situation de la qualité des eaux s'est en général stabilisée ou améliorée, sauf en milieu rural où elle s'est sensiblement dégradée et là où précisément l'agence Seine-Normandie intervient très peu : ce fait préoccupe ses instances qui ont voté le VIIème programme (19972001), en prévoyant des actions spécifiques en milieu rural.

Néanmoins ces indicateurs ne peuvent répondre correctement à la question posée, parce qu'on ne peut pas faire un lien simple et direct entre l'action financière de l'agence et l'évolution du milieu. Une des difficultés est que le cycle de vie de l'attribution des aides est de deux mois, celui des travaux est de 2 à 3 ans, celui de l'ouvrage varie de quelques années à plusieurs décennies, dans un contexte d'évolution technologique continuelle et de risques de défaillances toujours présents : comment relier tout cela au milieu naturel avec ses cycles de vie quotidien, saisonnier, pluriannuel, multiséculaire... et ses crises soudaines ? On retrouve ici une problématique récemment exposée aux Etats-Unis et relayée en France [19] sur des questions de prospective à long terme combinant des cycles lents et des cycles courts.

\section{2 - Faut-il désespérer ?}

"Nous ne sommes pas seuls au monde à chanter et le drame est l'ensemble des chants"8. L'agence n'est pas seule au sein de l'énorme système complexe représenté par l'impact des activités humaines sur les milieux aquatiques. La demande des présidents devrait être étendue pour évaluer à la fois l'efficacité de l'action réglementaire de l'Etat et eelle des maîtres d'ouvrage ; concernant ces derniers, de très récentes études montrent que certains prix de l'eau très faibles résultent en fait d'une inaction en matière d'équipement, de renouvellement et d'entretien d'installations existantes, la plupart du temps faute de provisions budgétaires à moyen terme.

Un problème préoccupant concerne les réseaux de mesures du milieu naturel : outre le fait qu'ils semblent inadaptés, l'aspect institutionnel n'a pas été clarifié [20]. Pourtant, depuis 1981, il avait été question de les confier à un "institut de l'eau" qui n'a pas encore vu le jour : cette

(6) Nos collègues de l'agence Loire-Bretagne ont fait une proposition analogue à leur conseil d'administration.

(7) Le territoire de Seine-Normandie est découpé en 57 zones hydrographiques qui pourront former chacune un Schéma d'Aménagement et de Gestion des Eaux (SAGE).

(8) Vers extrait d'"épilogue" dans "les poètes" de Louis Aragon 1960

Tableau 7. - Pollutions éliminées en 1994

(Extrait de la loi de finances de 1997 concernant Seine-Normandie).

\begin{tabular}{|c|c|c|r|}
\hline & Pollution domestique & MES en t/j & 1146 \\
Collectivités & et des industries raccordées, & MO en t/j & 511 \\
& éliminée par les stations & MA en t/j & 73 \\
& d'épuration urbaines & MP en t/j & 12 \\
\hline & & MES en t/j & 8436 \\
& Pollution éliminée & MO en t/j & 1448 \\
& par les industries & MA en t/j & 70 \\
& non raccordées & MP en t/j & 10 \\
& & MI en kilo Equitox par jour $(\mathrm{kEq} / \mathrm{j})$ & 24845 \\
\hline
\end{tabular}


idée a été reprise pour créer un autre organisme, l’Office International de l'Eau, auquel on a confié d'autres missions [21]. Cet état de fait est dommageable, car avec les réseaux de mesures et l'accès à leurs données, il serait possible de dynamiser la recherche sur les différents processus qui interviennent dans la fabrication de l'impact sur le milieu et la santé publique et de mieux comprendre l'action des différents intervenants. Tant que des décisions n'auront pas été prises dans ce domaine, il sera difficile de répondre scientifiquement à la question de l'évaluation et lorsqu'elles seront prises, il faudra sans doute beaucoup de temps, de transpiration et de patience pour y parvenir.

\section{RÉFÉRENCES}

[1] Comolet A. (1997), " Analyse rétrospective des programmes de l'AESN ". Actes du colloque de Montbard de juin 1996: " mesurer l'eau".

[2] LinSTER M. (1997). " Les indicateurs de l'OCDE ". Actes du colloque de Montbard de juin 1996 : " mesurer l'eau ".

[3] MOREL. B. (1997). "Les indicateurs de I'IFEN". Actes du colloque de Montbard de juin 1996 : " mesurer l'eau ".

[4] Le Moigne J.L. (1984). " La théorie du système général ; théorie de la modélisation ". PUF. 2ème édition mise à jour. 320 pages.

[5] LE MoIGNe J.L. (1990). " La modélisation des systèmes complexes ". Dunod. 178 pages.

[6] MORIN E. (1990). "Introduction à la pensée complexe". ESF éditeur. 158 pages.

[7] CÉRUtTI O. et Gattino B. (1992). " Indicateurs et tableaux de bord ". AFNOR : collection Gestion Qualité. 92 pages.

[8] Tardieu H., Nancy D., PAscot D. (1984) : "Conception d'un système d'information". Gaëtan Morin éditeur. 204 pages.
[9] Mélese J. (1991), “ L'analyse modulaire des systèmes (AMS) ”. Editions d'Organisation. 235 pages.

[10] TÉNIERE-Buchot P.F. (1989). " L'ABC du pouvoir ". Editions d'Organisation. 338 pages.

[11] Moles. A. (1990). “ Les sciences de l'imprécis ”. Seuil. 303 pages.

[12] Probst G.J.B., Ulrich H. (1989). “ Pensée globale et management. Résoudre les problèmes complexes ". Editions d'Organisation. 314 pages.

[13] SAOUT C. (1997). " La surveillance sanitaire des eaux ". Actes du colloque de Montbard de juin 1996: " mesurer l'eau ".

[14] Barbier J.M.( 1997). “ La production d'eau potable ”. Actes du colloque de Montbard de juin 1996 : " mesurer l'eau ".

[15] BORDET J.P. (1997). “ Exploitations statistiques ". Actes du colloque de Montbard de juin 1996: " mesurer l'eau ".

[16] MEYBeCK M. (1997), " Les réseaux de flux ”. Actes du colloque de Montbard de juin 1996: " mesurer l'eau ".

[17] DE MARSILY G. et FUSTEC E. (1995) “ Le programme CNRS PIRENSeine : une action de recherche puridisciplinaire et multipartenariale sur le fonctionnement global d'un bassin fluvial ". Rapport quadriennal du CNFGG, pages 237-246

[18] BALLAND P. (1997). “ Qu'est-ce qu'un réseau patrimonial "? Actes du colloque de Montbard de juin 1996 : " mesurer l'eau ".

[19] THEYs J.(à paraître). " Cycle de vie des politiques publiques de l'environnement et cycles naturels : une articulation problématique ". Actes du colloque de Fontevraud de septembre 1996 : “ quel environnement au XXIème siècle? ".

[20] TRUChot C.(1997). " Mesures et politique de l'Etat ". Actes du colloque de Montbard de juin 1996: " mesurer l'eau ".

[21] Preux D. (1997). “ Le système d'évaluation de la qualité de l'eau (SEQ-eau), le Réseau National de Données sur l'Eau (RNDE) et la banque de bassin ". Actes du colloque de Montbard de juin 1996 : " mesurer l'eau ". 